\title{
Investigations of concealed objects detection in visible, infrared and terahertz ranges
}

\author{
Marcin Kowalski, ${ }^{*}$ Mariusz Kastek, Norbert Palka, Henryk Polakowski, Mieczyslaw Szustakowski, Marek Piszczek \\ Institute of Optoelectronics, Military University of Technology, Warsaw, Poland
}

Received November 11, 2013; accepted December 16, 2013; published December 31, 2013

\begin{abstract}
Assuring the safety of crowded public places like e.g. airports is one of the most serious social issues. One of the aspects of the problem is the detection of potentially dangerous objects hidden under a person's clothing. There are various solutions from simple metal detectors to X-ray scanners. Some of the available solutions are harmful to humans. There is a great potential in using multispectral surveillance imagers in security systems thus we investigate the possibilities of concealed object detection by screening people with non-invasive cameras working in various spectral ranges.
\end{abstract}

The need to build highly efficient security systems for the detection of items covered with fabrics is a very urgent topic for researchers. This problem is particularly important for the safety of airport passengers. One of the solutions is a terahertz $(\mathrm{THz})$ imager [1]. Practical, realtime imaging in the terahertz band is of great interest for the screening of people, particularly the detection of dangerous objects at the standoff range [2-3]. The terahertz waves offer great possibilities in the field of concealed objects detection because they can penetrate various materials and are not harmful to humans [4-5]. However, the main difficulty in the $\mathrm{THz}$ imagers is low spatial resolutions and low image quality [6].

Another possible solution is a high resolution infrared camera. Because of the fact that infrared cameras can detect temperature differences on the surface of an object, it is justified to investigate the possibilities of applying this type of imagers for detection of hidden items [7-8].

The measurement setup consists of four cameras, infrared imaging Fourier-transform spectro-radiometer (IFTS) [9], two thermoelements and thermo-higrobarometer. The four cameras cover a very wide range of spectra - from visible light up to terahertz. There are four cameras - a visible light (VIS) camera, passive terahertz $(\mathrm{THz})$ camera and two infrared cameras. All of the devices are commercially available. A photographic fabric was used as a background of the measurement scene. The measurement setup is presented in Fig. 1.

The $\mathrm{THz}$ camera employed in the measurements is the TS4 camera from ThruVision operating at $250 \mathrm{GHz}$ with a resolution of $124 \times 271$ pixels [10]. Another camera is a long-wavelength infrared (LWIR) camera P640 from

\footnotetext{
*E-mail: mkowalski@wat.edu.pl
}

FLIR supplied with an uncooled microbolometer detector working in the range of $7.5-13 \mu \mathrm{m}$ and with a resolution of $640 \times 480$ pixels. The thermal sensitivity of the LWIR camera is $55 \mathrm{mK}$ at $30^{\circ} \mathrm{C}[8]$.

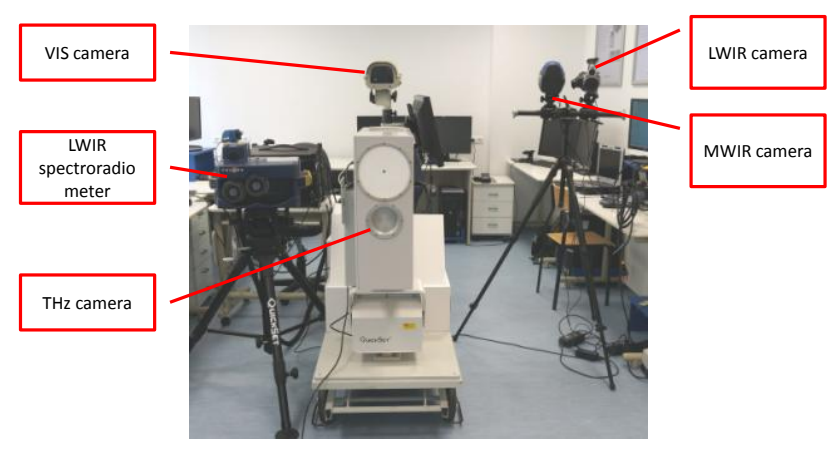

Fig. 1. Measurement setup.

The second infrared camera is a mid-wavelength infrared (MWIR) SC5600 camera from FLIR. The camera is supplied with a cooled microbolometer detector (3$5 \mu \mathrm{m})$ with a resolution of $640 \times 480$ pixels. The thermal sensitivity of the MWIR camera is typically $20 \mathrm{mK}$ [8]. Complementary images acquired with the four cameras are presented in Fig. 2.

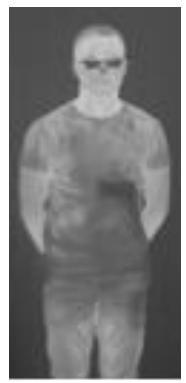

(a)

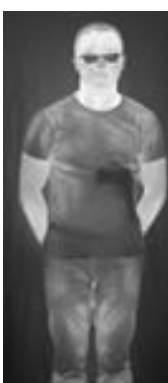

(b)

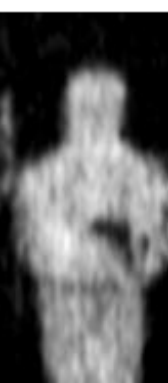

(c)

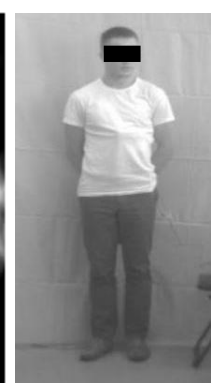

(d)
Fig. 2. Complementary images: (a) LWIR image, (b) MWIR image, (c) THz image, (d) VIS image.

The infrared imaging Fourier-transform spectrometer Hyper-Cam LWIR use $320 \times 256$ pixel Mercury Cadmium Telluride (MCT) focal plane arrays (FPA) with a $6^{\circ} \times 5^{\circ}$ FOV. Spectral information is obtained using a technique 
called Fourier Transform Infrared Radiometry (FTIR). FTIR is a classical interference based technique applied to gas spectroscopy that uses a Michelson interferometer to mix an incoming signal with itself at several different discrete time delays. The IFTS collect datacubes (discrete infrared thermogram for a wave number) which enables to calculate spectral signatures of clothes, weapons, etc [11].

Due to the fact that the thermal camera measures the relative temperature of objects and because, during the measurements, an object is covered with a material, it measures the temperature of the surface of a covering material. A thermal camera can be used to detect an object covered with a fabric only in certain conditions. The main condition is the value of a temperature difference between an object and the surface of a covering material. In order to detect the temperature difference, the thermal sensitivity of an infrared imager should be as low as possible [8].

The radiation distribution detected by the passive terahertz imager is proportional to the relative temperature of a target and is directly related to the absolute amount of $\mathrm{THz}$ radiation emitted by the target.

During the measurements, the hidden object is heated by a human body, therefore the value of temperature difference between a covering material and an object may decrease. This fact should be considered in order to evaluate the potential of infrared and terahertz cameras to detect a hidden object.

The organization of measurements is presented in Fig. 3. During the measurements, several configurations with various objects (guns, knives, dynamite) and clothes (shirt, T-shirt, sweater) were prepared. The measurement of one single configuration took 30 minutes. A set of data from cameras (four images), spectroradiometer and thermoelements were collected every five minutes. For every five minutes, the data package consists of images, spectral signatures collected in LWIR range, values of atmosphere parameters (air temperature, humidity and pressure) and values of body and object temperatures.

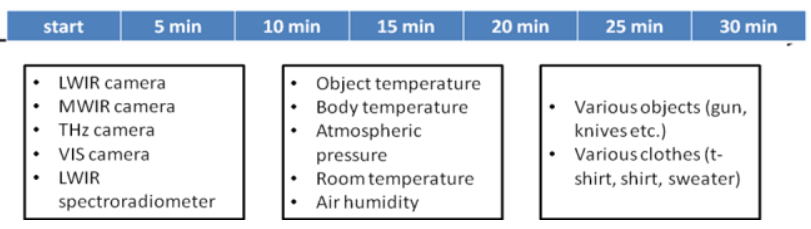

Fig. 3. Measurement organization.

A set of images acquired with four cameras $-\mathrm{THz}$, LWIR, MWIR and VIS with single test object (a gun) and one type of clothing (T-shirt) is presented in Tab. 1. It should be noticed that the measurement background was uniform.
Tab. 1. Initial results of investigations
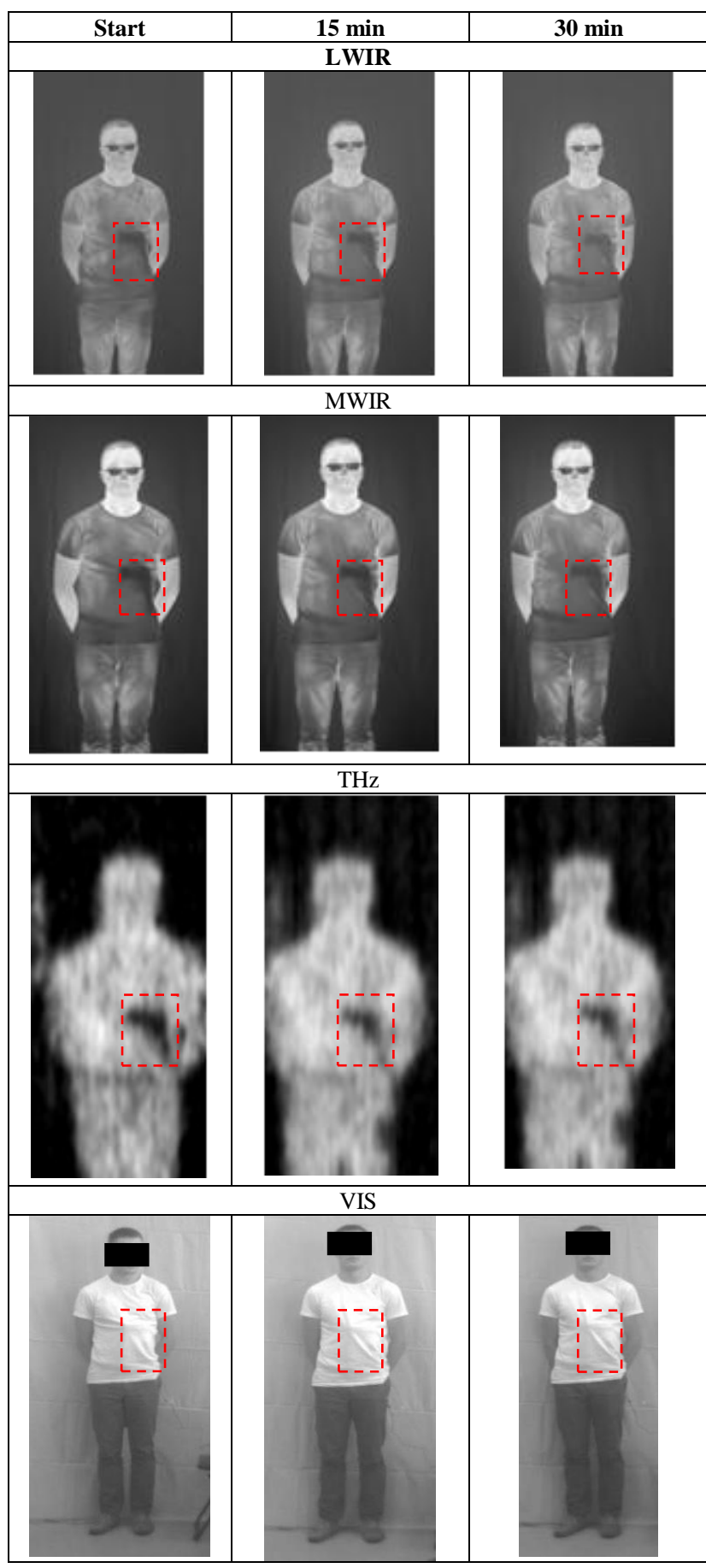

Subjectively, the location of a hidden object is visible in every image except the images acquired with a visible light camera. It can be noticed that the visibility of the concealed object decreases with time. It results from a decrease in the temperature difference between the object and the human body. It should be noted that the ability to detect a concealed object with a camera depends on the type of clothing material and the thickness of clothing. 
Comparing the images registered with two thermal imagers it can be noticed that the hidden object is more distinct in the images registered with the MWIR camera.

However, to evaluate the capabilities of an imager to detect a hidden object, it is justified to process images in order to extract the hidden object. Image processing is an inevitable element of any surveillance security system. In the case of a system for detecting concealed objects, the system operator should be provided with clear and understandable information [8, 12]. The examples of binary images are presented in Fig. 4.

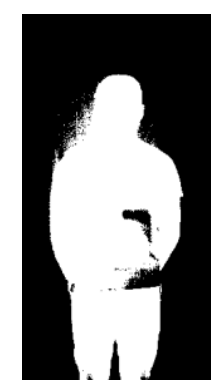

(a)

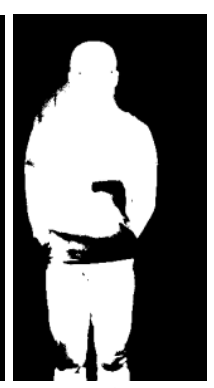

(b)

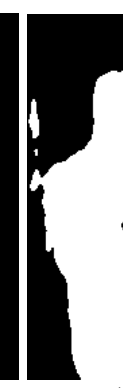

(c) values of temperature measured with thermoelements and the values marked blue present the values of relative temperature measured with thermal MWIR imager. The emissivity of clothes and weapons were measured using the spectrometer. The value of emissivity of clothes was between $0.92 \pm 0.03$ in the MWIR range, and $0.94 \pm 0.02$ in the LWIR range. The results were used during the calculations of temperatures. The differential temperatures are presented in Fig. 6b. It can be noticed that the differential temperatures measured with the two methods are different. This can be due to the fact that the thermal camera measures the radiation temperature of clothing this is not a direct measurement of the temperature of an object.

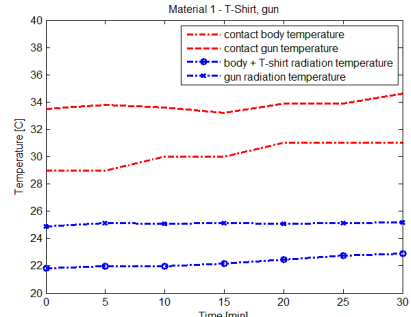

(a)

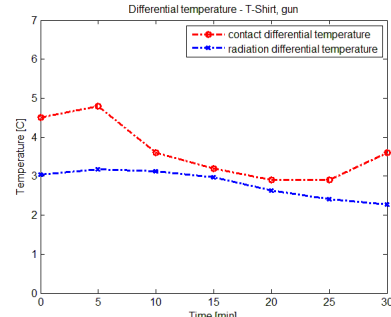

(b)
The processed images presented in Fig. 4 show the possibilities of extracting a detected object. The processed VIS image presented in Fig. 4(d) confirms the fact that it is not possible to detect an concealed object with a single visible light camera. However, the VIS image can be utilized in the image fusion process [12-14]. The processed thermal and terahertz images presented in Fig. 4(a)-(c) show the detected object. However, the image processing algorithms need to be improved because of the artifacts remaining in the images after processing.

It should be noticed that the measurement conditions were controlled. In Fig. 5, two regions of the thermograms were marked. These regions were selected to measure the mean values of temperature.

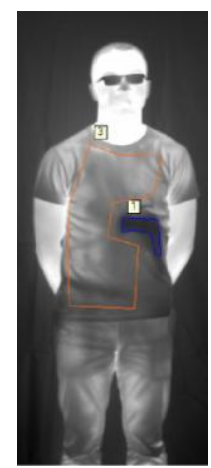

Fig. 5. Thermogram registered with MWIR camera with selected regions of interests

The values of measured temperatures are presented in the graph in Fig. 6a. The values marked red present the
Fig. 6. Temperatures of body and gun during measurements; (a) real temperatures, (b) differential temperatures.

The results presented in the article show how the changes of temperature values of a human body and an object can influence the ability to detect the concealed object. The results confirm the fact that it is possible to detect objects (gun) covered with a T-shirt placed on a human body using a thermal and terahertz imager in certain conditions. The parameters of imagers are essential for long lasting detection of objects. The results indicate that it is not possible to detect a concealed object with a single visible light camera.

\section{References}

[1] C. Jansen et al., Appl. Optics 19, 49 (2010).

[2] M.C. Kemp, Proc. SPIE 6402, 64020D (2006).

[3] E. Grossman et al., Appl. Optics 19, 49 (2010).

[4] L. Yun-Shik, Principles of Terahertz Science and Technology (Springer 2008).

[5] X. Zhang, J. Xu, Introduction to THz Wave Photonics (Springer 2010).

[6] B.B. Hu, M.C. Nuss, Opt. Lett. 20, 16 (1995). (2011).

[8] M. Kastek et al., Proc. SPIE 8382, 83820K (2012).

[9] M. Kastek et al., Phot. Lett. Poland 4, 4 (2012).

[10] http://www.digitalbarriers.com/products/thruvision/

[11] M. Kastek, T. Piątkowski, P. Trzaskawka, Metr. Measur. Sys. 18, 4 (2011).

[12] M. Kowalski, N. Palka, M. Piszczek, M. Szustakowski, Acta Phys. Pol. A 124, 3 (2013).

[13] M. Kowalski, N. Palka, M. Piszczek, M. Szustakowski, Phot. Lett. Poland 4, 3 (2012).

[14] M. Kowalski, N. Palka, M. Piszczek, M. Szustakowski, Phot. Lett. Poland 5, 1 (2013)
[7] M. Piszczek , M. Kowalski, M. Szustakowski, Acta Phys. Pol. A 4, 120 